\title{
Impact of surveillance frequency on survival in non-small cell lung cancer (NSCLC) survivors
}

\author{
Jyoti Malhotra, Salma K. Jabbour, Sharon Pine \\ Rutgers Cancer Institute of New Jersey and Robert Wood Johnson Medical School, New Brunswick, NJ, USA \\ Correspondence to: Jyoti Malhotra, MD, MPH. Rutgers Cancer Institute of New Jersey, Robert Wood Johnson Medical School, 195 Little Albany \\ street, New Brunswick, NJ 08903, USA. Email: jm1940@cinj.rutgers.edu. \\ Provenance: This is an invited article commissioned by the Section Editor Hengrui Liang (Department of Thoracic Surgery, Guangzhou Medical \\ University, Guangzhou, China). \\ Comment on: McMurry TL, Stukenborg GJ, Kessler LG, et al. More Frequent Surveillance Following Lung Cancer Resection Is Not Associated \\ With Improved Survival: A Nationally Representative Cohort Study. Ann Surg 2018;268:632-9.
}

Submitted Apr 23, 2019. Accepted for publication May 07, 2019.

doi: $10.21037 /$ tlcr.2019.05.04

View this article at: http://dx.doi.org/10.21037/tlcr.2019.05.04

Lung cancer is the second most common cancer in both men and women and non-small cell lung cancer (NSCLC) accounts for more than $86 \%$ of all lung cancers (1). Lung cancer survivors are a growing yet understudied patient population. Even with the high mortality associated with lung cancer, the number of lung cancer patients added to the survivor population every year is significantly high due to high lung cancer incidence rates $(224,210$ new cases diagnosed annually) (1). Approximately 26,000 individuals become long-term lung cancer survivors annually (2). There are approximately 526,510 lung cancer survivors in the US as of January 2016, and this number is expected to rise to 673,370 by 2026 (1). Beyond this estimate, the number of survivors will likely increase further with the implementation of lung cancer screening in smokers that can detect patients with early-stage (stage I and II) NSCLC. The majority of the lung cancer survivors are patients with early-stage NSCLC who are treated with surgery. These patients have a significant risk of mortality as the 5-year survival for early-stage NSCLC is only 55.6\% (3). These poor survival rates are primarily because lung cancer survivors have a significantly higher risk for developing another lung cancer; either a new cancer (1-2\% per-year risk) or recurrent disease (10-38\% overall risk) $(4,5)$. They are also at higher risk for other cancers such as cancers of the larynx, colon, esophagus and stomach with the estimated risk being 3-6\% per year (6).

Due to the high mortality in lung cancer survivors, early detection and treatment of both recurrence and second cancers in these patients is paramount to improved outcomes. A study in 1,294 patients with lung cancer found a $20 \%$ recurrence rate and a $7 \%$ rate for second primary cancers with median follow-up of 35 months from diagnosis. Approximately $93 \%$ of second cancers and $61 \%$ of recurrences were identified by surveillance scans (7). Early identification of recurrence or a second cancer through surveillance may improve survival. In a prospective study in 192 lung cancer survivors, the 3-year survival was improved in the 36 patients whose asymptomatic recurrence was identified by surveillance (31\%) compared to $13 \%$ in all patients (8). In a retrospective analysis using SEERMedicare linked database in 10,680 patients with earlystage NSCLC treated with surgical resection, survivors who received regular imaging experienced improved survival compared to survivors who did not (9). While there is evidence to support regular surveillance, there is no consensus on the optimal frequency and timing of surveillance after curative resection of lung cancer. Clinical guidelines from the National Comprehensive Cancer Network (NCCN) as well as the European Society for Medical Oncology (ESMO) recommend CT scans every 6 months for first 2-3 years after lung cancer resection and yearly CT scans thereafter $(10,11)$. However, these clinical guidelines are consensus-based and not evidence-based as there is not enough data to define the optimal strategy for surveillance in these patients. 
In 2018, McMurry et al. reported results from a retrospective analysis of the National Cancer Database (NCDB) that investigated the association between intensity of surveillance and survival following surgical resection for NSCLC (12). This study randomly selected patients who were diagnosed with Stage I to III NSCLC between 2006 and 2007 and underwent surgical resection. A total of 4,463 patients were included and grouped into three surveillance groups based on time from surgery to first surveillance using CT scan (3-, 6-month and annual). Patients were followed from the time of surgery through December 2012 or until first recurrence, new second cancer or death. Analysis was restricted to patients who remained disease free through 14 months postoperatively $(\mathrm{n}=3,552)$. Approximately $11.0 \%$ of these patients developed a new second cancer and $23.8 \%$ developed a recurrence during the follow-up period. There was no difference in these rates between the surveillance groups $(\mathrm{P}$ value $=0.49)$. In this cohort, more frequent surveillance was not associated with longer risk-adjusted overall survival. The hazard ratio (HR) for 6-month followup relative to 3 months was 1.16 [95\% confidence interval (CI): 0.99 to 1.36], whereas the HR for yearly surveillance compared to 3 months was 1.06 (95\% CI: 0.86 to 1.31 ). The authors concluded that more frequent surveillance after lung cancer surgery is not associated with improvement in survival.

The study by McMurry et al. (12) described above has a number of notable strengths. The analysis utilized a unique dataset through a Commission on Cancer $(\mathrm{CoC})$ special study mechanism that augmented data from NCDB. This $\mathrm{CoC}$ initiative was part of a quality improvement effort so that there is better documentations of comorbidity and recurrence of NCDB data and as a result, this study had access to data on recurrence abstracted by registry staff from the patient records. The dataset is also representative of the general population as NCDB captures data from about $70 \%$ newly diagnosed cancer cases from more than 1,500 hospitals in the United States (13). Also, only 4\% patients had missing information about treatment or comorbidities. However, the main limitation of this study is the retrospective nature and inclusion of patients with stage III NSCLC. The only variable that was significantly different between the three surveillance groups was the stage of NSCLC. The frequent surveillance (3-month) group had more patients with stage III disease $(18.7 \%$ vs. $15 \%)$ and fewer patients with stage I disease ( $60 \%$ vs. $65 \%)$ compared to the 6-month and annual surveillance groups. As patients with more advanced stage have worse survival, this may have introduced bias in the results. It would have been interesting to see the impact on the study findings if the analysis was stratified by stage. A retrospective study using SEER-Medicare merged dataset reported that receipt of surveillance scan between 4 to 8 months after surgery in patients with NSCLC was associated with improved survival in stage I NSCLC (HR: 0.85 ; $95 \%$ CI: 0.74 to 0.98 ), but not in stage II (HR: 1.01; 95\% CI: 0.71 to 1.42 ) (14).

The frequency of surveillance in early-stage NSCLC survivors is an important question which warrants a prospective study. Any retrospective analysis looking at this question (including the study by McMurry et al.) will be complicated by two factors: selection bias due to excluding patients who recur or are lost to follow-up and poor health utilization by lung cancer survivors. For instance, McMurry et al. excluded patients who recurred or died in the first 14 months after surgical resection so that they can balance the three surveillance groups (3- vs. 6-month vs. annual) and have complete follow-up data for patients in the three groups. However, a complication of this methodology was that patients who had early recurrence (within 1 year) and would have benefitted most from frequent surveillance were excluded from the analysis. Moreover, lung cancer survivors have the lowest score in health care utilization when compared to long-term survivors of all other cancers (15). Therefore, even if more frequent surveillance detects an early recurrence or second primary, this may not translate into earlier treatment initiations due to poor health care utilization by lung cancer patients. A SEER-Medicare analysis in patients with stage I NSCLC diagnosed between 1998 and 2008 reported that only $61.4 \%$ of patients received surveillance in the first 2 years after treatment (16). A number of factors impact health care utilization in lung cancer survivors such as age, geographic location, race/ ethnicity and comorbidities (9).

Most studies to date investigating surveillance strategy in lung cancer survivors are retrospective and use data from patients diagnosed before $2010(9,12,16)$. Over the last decade, a number of novel therapies have become available for NSCLC treatment. This includes targeted therapy, immunotherapy as well as improved safety and recovery with minimally invasive surgical techniques. A recent metaanalysis reported that robotic lobectomy for the treatment of lung cancer is associated with less complications and lower 30-day mortality compared to open surgery (OR: 0.53 ; $95 \%$ CI: 0.33 to 0.85 ) (17). With the advent and adoption of these therapeutic advances, survival rates for lung cancer are improving. Even for patients with 
metastatic or stage IV NSCLC, the long-term survival rate has improved from less than $4 \%$ to $14 \%$ with patients who respond to immunotherapy having a 4-year survival as high as $58 \%$ (18). The role of surveillance and associated benefits over the last few years needs to be investigated as patients with earlier identification of recurrence or new primary may have better outcomes due to improved treatment options that are available.

In conclusion, the results from McMurry and colleagues report a lack of benefit from more frequent (at 3 months compared to 6 or 12 months) surveillance in patients treated for early-stage NSCLC with surgical resection. The optimal strategy for surveillance in these survivors has not been clear as studies investigating this to date including the study by McMurry et al. are limited by factors such as retrospective nature, selection bias, and lack of data on patients diagnosed in the last 10 years. Prospective research in this area is warranted as lung cancer continues to be associated with significant morbidity and mortality.

\section{Acknowledgments}

None.

\section{Footnote}

Conflicts of Interest: The authors have no conflicts of interest to declare.

\section{References}

1. Siegel RL, Miller KD, Jemal A. Cancer statistics, 2015. CA Cancer J Clin 2015;65:5-29.

2. Jemal A, Thun MJ, Ries LA, et al. Annual report to the nation on the status of cancer, 1975-2005, featuring trends in lung cancer, tobacco use, and tobacco control. J Natl Cancer Inst 2008;100:1672-94.

3. SEER*Stat Database: Incidence - SEER 18 Regs Research Data + Hurricane Katrina Impacted Louisiana Cases, Nov 2014 Sub (1973-2011 varying), National Cancer Institute, DCCPS, Surveillance Research Program, Surveillance Systems Branch. [database on the Internet]. Available online: https://seer.cancer.gov

4. Pepek JM, Chino JP, Marks LB, et al. How well does the new lung cancer staging system predict for local/regional recurrence after surgery? A comparison of the TNM 6 and 7 systems. J Thorac Oncol 2011;6:757-61.

5. Hamaji M, Allen MS, Cassivi SD, et al. Surgical treatment of metachronous second primary lung cancer after complete resection of non-small cell lung cancer. J Thorac Cardiovasc Surg 2013;145:683-90; discussion 690-1.

6. Surapaneni R, Singh P, Rajagopalan K, et al. Stage I lung cancer survivorship: risk of second malignancies and need for individualized care plan. J Thorac Oncol 2012;7:1252-6.

7. Lou F, Huang J, Sima CS, et al. Patterns of recurrence and second primary lung cancer in early-stage lung cancer survivors followed with routine computed tomography surveillance. J Thorac Cardiovasc Surg 2013;145:75-81; discussion 81-2.

8. Westeel V, Choma D, Clement F, et al. Relevance of an intensive postoperative follow-up after surgery for non-small cell lung cancer. Ann Thorac Surg 2000;70:1185-90.

9. Malhotra J, Rotter D, Jabbour SK, et al. Receipt of recommended surveillance with imaging in elderly survivors of early stage non-small cell lung cancer. Lung Cancer 2018;125:205-11.

10. Vansteenkiste J, Crino L, Dooms C, et al. 2nd ESMO Consensus Conference on Lung Cancer: early-stage nonsmall-cell lung cancer consensus on diagnosis, treatment and follow-up. Ann Oncol 2014;25:1462-74.

11. Non-small cell lung cancer (Version 2.2019). National Comprehensive Cancer Network.

12. McMurry TL, Stukenborg GJ, Kessler LG, et al. More Frequent Surveillance Following Lung Cancer Resection Is Not Associated With Improved Survival: A Nationally Representative Cohort Study. Ann Surg 2018;268:632-9.

13. Bilimoria KY, Stewart AK, Winchester DP, et al. The National Cancer Data Base: a powerful initiative to improve cancer care in the United States. Ann Surg Oncol 2008;15:683-90.

14. Backhus LM, Farjah F, Liang CK, et al. Imaging surveillance and survival for surgically resected non-smallcell lung cancer. J Surg Res 2016;200:171-6.

15. Sugimura H, Yang P. Long-term survivorship in lung cancer: a review. Chest 2006;129:1088-97.

16. Erb CT, Su KW, Soulos PR, et al. Surveillance Practice Patterns after Curative Intent Therapy for Stage I NonSmall-Cell Lung Cancer in the Medicare Population. Lung Cancer 2016;99:200-7.

17. O'Sullivan KE, Kreaden US, Hebert AE, et al. A systematic review and meta-analysis of robotic versus open and video-assisted thoracoscopic surgery approaches for lobectomy. Interact Cardiovasc Thorac 
Surg 2019;28:526-34.

18. Brahmer J, Borghaei H, Ramalingam SS, et al. Long-term survival outcomes with nivolumab (NIVO) in pts with previously treated advanced non-small cell lung cancer

Cite this article as: Malhotra J, Jabbour SK, Pine S. Impact of surveillance frequency on survival in non-small cell lung cancer (NSCLC) survivors. Transl Lung Cancer Res 2019;8(Suppl 4):S347-S350. doi: 10.21037/tlcr.2019.05.04
(NSCLC): Impact of early disease control and response. Available online: https://www.abstractsonline.com/ pp8/\#!/6812/presentation/9964 